\title{
Conversational agents in online organization-stakeholder interactions: a state-of-the-art analysis and implications for further research
}

\author{
Salla Syvänen and Chiara Valentini \\ Jyväskylä University School of Business and Economics, Jyväskylä, Finland
}

\begin{abstract}
Purpose - The purpose of this study is to review the extant literature on chatbots and stakeholder interactions to identify major trends and shed light on knowledge gaps.

Design/methodology/approach - A systematic literature review was conducted combining qualitative and quantitative approaches. A code book based on early systematic literature reviews was developed and used to extract information from 62 discrete peer-reviewed English articles. An inductive approach was used to analyse definitions of chatbots, topics, metrics, perspectives and implications.

Findings - Chatbots have been studied by many different disciplines, but not much from organizational, stakeholder and corporate communication perspectives. Existing studies focus on the technical developments of chatbots and chatbot language and conversations skills. Research has remained anchored at the micro-level understanding of the phenomenon, that is, the nature of chatbots, but has not yet taken into consideration the meso (organizational) or macro (societal) levels.

Research limitations/implications - This study focused only on academic peer-reviewed papers in English and excluded conference proceeding, books, book chapters and editorials that may have offered other important and relevant reflections. The limited number of studies in communication-related disciplines shows that corporate communication scholars could contribute more to the discussion of chatbot-stakeholder interactions.

Originality/value - This is the first research in the field of corporate communication that examines organizational chatbot-stakeholder interactions. Results of this review offer important information on chatbots' organizational capabilities and affordances, which, arguably, must be taken into consideration when stakeholder engagement strategies are set.
\end{abstract}

Keywords Chatbot, Systematic literature review, Corporate communication, Artificial intelligence,

Organization-stakeholder interactions

Paper type Literature review

\section{Introduction}

The twenty-first century has been characterized by an increased digitalization of processes and activities. Human-robot interactions are less and less fiction and more and more common encounters with organizations seeking to maximize their capabilities (Galloway and Swiatek, 2018). From a corporate communication perspective, human-robot interactions as part of artificial intelligence (AI) can offer a number of opportunities, for instance, improving the way information is delivered (i.e. via augmented reality and virtual reality apps) and providing better insights and predictive analytics for decision making (Petrucci, 2018, April 20).

(C) Salla Syvänen and Chiara Valentini. Published by Emerald Publishing Limited. This article is published under the Creative Commons Attribution (CC BY 4.0) licence. Anyone may reproduce, distribute, translate and create derivative works of this article (for both commercial \& non-commercial purposes), subject to full attribution to the original publication and authors. The full terms of this licence may be seen at http://creativecommons.org/licences/by/4.0/legalcode

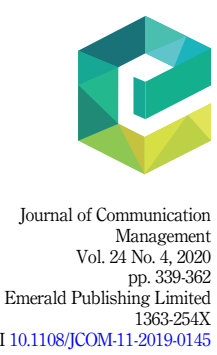

Agents in organizationstakeholder interactions 
$\mathrm{JCOM}$ 24,4

However, the use of AI for managing online stakeholder relations has created new challenges for corporate communication professionals, not just because of the complexity of many relational tasks, but also because of the impact that using such technologies can have on developing and/or maintaining trustworthy relationships. When technologies do not correctly handle complex requests (Galloway and Swiatek, 2018; Tilson, 2017), organizations may experience negative forms of engagement (Lievonen et al., 2018).

This study was interested in a specific type of AI, chatbots, in the context of online interactions between stakeholder and organizations. Chatbots are computer programmes designed to simulate conversations with human users, especially on the internet (Oxford Dictionary, 2019). Recent studies have shown that organizations across industries are employing chatbots, often as a tool for customer services (Bingjie and Sundar, 2018), and that stakeholders seem to hold a positive attitude towards chatbots (Hill et al., 2015; De Kleijn et al., 2019). The AI field, particularly with regards to chatbots, is in continuous development; therefore, it is hard to predict the extent to which these technologies are capable of addressing elaborate tasks beyond specific customer-related ones, such as answering inquiries, offering information and so on. Yet, because they are becoming a common interface between stakeholders and organizations, it is necessary to reflect on the opportunities and threats that these technologies offer with respect to corporate communication goals, such as building mutual and beneficial relationships with stakeholders and publics (Grunig and Huang, 2000). After all, AI platform design is purposeful and exhibits biases that may erode human rights (Bourne, 2019).

Thus, the purpose of this study was to conduct a systematic literature review to assess the level of academic knowledge regarding chatbot-stakeholder interactions as a first step in assembling and assessing existing knowledge in a field where little is known. In the following sections, the conceptual foundations of this study are discussed followed by a presentation of the research questions, methodology and findings. The paper concludes with a discussion regarding the current status of research on chatbot-stakeholder interactions and offers suggestions for future research.

\section{Context and conceptual foundations}

\section{Maintaining online mediated stakeholder interactions}

The body of knowledge in online public relations has primarily focused on human-tohuman interactions via social or digital media for different purposes and situations and in different contexts - private, public and non-profit organizations (cf. Duhé, 2015). In this area, some studies examined the agency role of digital channels to identify new ways to build and maintain organization-stakeholder relationships, for instance, those on microblogging networks (Chen and $\mathrm{Fu}, 2016$; Wang et al., 2018). Especially informative posts were found to be most shareable and to increase engagement (Chen and $\mathrm{Fu}, 2016$; Gao et al., 2014). Being active in online and social media communities can improve company brand differentiation and strengthen relationships with customers, but it also positively impact customer engagement through social relatedness and trust (Ji et al., 2017; Kim and Drumwright, 2016; Liu et al., 2018; Martinez-Lòpez et al., 2017; Rybalko and Seltzer, 2010).

Overall, social media has become an essential part of organization communication strategies but dialogic capabilities of social media are limitedly utilized. Stakeholder participative expectations are often not met because organizations tend to hesitate in deploying dialogic communications (Huang and Yang, 2015; Navarro et al., 2017; Watkins, 2017) and instead, prefer to use them for pushing contents that support their corporate reputation (Wang et al., 2016). While research focusing on social media is contradictory regarding its actual value for stakeholder engagement (Simon and Tossan, 2018), specific 
studies, for instance, on social messengers (Tsai and Men, 2018), found that they offer opportunities for organizations to achieve a strong one-on-one connection with individual stakeholders. Social messengers can be efficient tools for maintaining human-to-human interactions, but AI has brought new venues for interacting with humans. AI conversational bots have been tested and used in instant messenger platforms, and results of diverse experiments and usage are promising (e.g. Goh et al., 2008). However, researchers are cautious as there are still differences in the quality and content of these conversations when compared to human-to-human interactions (Hill et al., 2015).

This study focused on a specific type of AI, chatbots, in the context of online interactions between stakeholders and organizations. The history of the chatbot begins in 1966 when Joseph Weizenbaum developed ELIZA, "a computer program for the study of natural communication between man and machine" (Weizenbaum, 1966). Today, chatbots have become an important tool for customer services (Bingjie and Sundar, 2018), but other usages are possible since chatbots can process a large amount of data independently and can learn to perform new tasks.

\section{Organizational capabilities of chatbots}

To better understand the possible usage of chatbots, it is necessary to reflect upon the function of chatbots in an organizational context. Assuming that chatbots provide new organizational capabilities, this section presents and discusses the most important ideas in the literature of organizational capabilities. Management research offers an incredible number of studies on organizational capabilities as intangible organizational resources offering competitive advantage. Organizational capabilities are generally understood as a sum of organizational competencies and individual abilities that turn know-how into results (Ullrich and Smallwood, 2004). Capabilities thus emerge from the advancement of organizational competences and routines and entail an integration of specialist knowledge across units, departments and individual members (Teece and Pisano, 1994). Particularly, the routinization of organizational activities facilitates the retention of capabilities into organizational memory, which, in turn, provokes a distinctive configuration of organizational resources (Knight and Cavusgil, 2004). Organizational capabilities have been found, for example, to predict the ability of an organization to internationalise (McDougall et al., 1994), to innovate (Knight and Cavusgil, 2004) and to retain its best employees (Gilani and Cunningham, 2017).

Organizational capabilities can have many sub-forms. In relation to chatbots, it is relevant to discuss technological capabilities, which refers to the broader context of technologies in organizations (Bharadwaj, 2000) and often describes those specific capabilities related to the effective use of technological knowledge "to assimilate, use, adapt and change existing technologies" (Dutrénit, 2004, p. 210), but also includes the capacity to develop new technologies or new products and processes (Kim, 1997). Technological capabilities have been considered central to the process of increasing productivity leading to greater organizational competitiveness (Bell and Pavitt, 1995). It has been noted that computers are more and more resembling humans in their abilities in performing tasks and in their capacity to learn while operating, such as dealing with matters from the office and home environment to routine purchases to strategic organizational decisions (Carley, 2002). It is exactly the capacity to analyse, perform, deliver and routinize that make them an important organizational capability.

Chatbots are a particular type of technology in that they show characteristics of autonomy and independent learning, which makes them more like "smart agents (Carley, 2002) because they engage in cognitive processing of information to respond to stimuli. Furthermore, research on artificial intelligence shows that smart agents can impact organizations on three levels: organizational boundaries, communication and storage (Carley, 2002). Smart agents 
$\mathrm{JCOM}$ 24,4

\section{2}

can permeate the boundaries between people, tasks and resources, as they break the distinction not just of internal versus external boundaries, but also between different actors and objects in, out and among organizations. Smart agents act as communicators on behalf of organizations. Thus, the accuracy, the truthfulness and frequency of their communication has an impact on organizational reputation and performance. Smart agents can learn and retain a great deal of information and develop new knowledge that is not necessarily shared with humans; thus, they can affect group knowledge and organizational memory (cf. Carley, 2002 for a detailed discussion). Research in human-computer interaction has shown that people interacting with AI technologies perceive them as distinct social actors rather than technologies (Nass et al., 1994), confirming thus that AI is more and more positioned as "increasingly complex and life-like communication partners" (Guzman and Lewis, 2020, p. 4). Research in organization-stakeholder relationships has primarily focused on interpersonal communication dynamics and more recently, in mediated forms of online relationships, which are still forms of human-to-human communication.

\section{Affordances in communication studies}

Given the very nature of chatbots and their capabilities, one must reflect upon the nature of its agency and what it entails to do. Chatbots can exercise agency through their "performativity" (Barad, 2003; Pickering, 1995), or "through the things they do that users cannot completely or directly control" (Leonardi, 2011, p. 144). Yet, chatbot agency can be measured in terms of affordances. There is a plethora of definitions of affordances, but for the most part, they are described as possibilities for action. Typically, an affordance refers to a particular kind of functionality of a technology (Faiola and Matei, 2010), but it is not just a material property of a technology, it can also be a relational one. This means that the property is constructed in the interaction between people and technology (Hutchby, 2001). Affordances are dynamic and always emerging from the relationship between people and the environment. They can have both positive and negative, intended and unintended and short- and long-term associations; they may both enable and constrain actions (Conole and Dyke, 2004; Majchrzak et al., 2013).

New affordances may emerge because of the development of new technologies and this development may offer new possibilities for action by individuals (Evans et al., 2017). A recent study by Stoeckly et al. (2018) explored affordances of organizational chatbots in the context of enterprise systems and found 14 functional affordances that were later categorised into four groups. The identified affordances essentially represent properties of chatbots as objects in the studied organizations. These properties relate to the capacity of chatbots: "(1) to facilitate alignment by integrating information (e.g., receiving messages), (2) to provide control mechanisms (e.g., getting and setting triggers), (3) to enable interoperability (e.g., querying and invoking functionality of thirdparty systems, and (4) to increase efficiency (e.g., enriching messages)" (Stoeckly et al., 2018, p. 2023). Yet, the literature on affordances has shown that there are various understandings and perspectives. Affordances can be properties of the object/ environment, a latent capability emerging in a particular context, or specific to the actor/species (Rice et al., 2017, p. 108).

\section{Methodology}

The scope of this study was to examine how chatbots have been studied to learn more about the opportunities and constraints they may offer to corporate communication; qualitative and quantitative approaches were employed (cf., White et al., 2017; Lock, 2019). The systematic review was driven by the following research questions: 
$R Q 1$. How are chatbots defined and used in the context of organization-stakeholder interactions?

RQ2. What are the major theoretical and methodological approaches used to study chatbot exchanges?

RQ3. What are the major perspectives and implications discussed in relation to chatbots?

The following sections explain the protocol, data selection, extraction and analysis.

\section{Data collection procedures}

Since the topic of interest is interdisciplinary in nature, a diversity of epistemological approaches and disciplinary traditions were included. The analysis was restricted to English language articles published in the established databases, ProQuest, EBSCO and CMMC. The search was open to all peer-reviewed articles, excluding conference proceedings, books, book chapters and editorials (White et al., 2017; Ye and Ki, 2012). Because the chatbot is a new phenomenon, the search criteria had no time constraints. To identify relevant articles, a string of keywords comprising a combination of "chatbot" or "bot" or "artificial intelligence" or "human-robot" or "human-computer" and "dialogue" and/or "conversation" and/or "interaction" and/or "discussion" and/or "discourse" and/or "relationship" was used in the abstract and title. The search was initiated on 31 January 2019 and completed on 11 October 2019.

The search resulted in 112 articles, which were further screened to remove duplicates and irrelevant papers. As the focus of this study was on interactions between humans and chatbots, all articles were removed from the data set that dealt with social bots or social or human robots without a clear chatbot connection. The final sample consisted of 62 discrete articles.

\section{Data extraction and analysis}

Based on early literature reviews (Lock, 2019; White et al., 2017; Ye and Ki, 2012), a coding protocol was created and shared between the two independent coders (see Appendix 1). Articles were reviewed by extracting the following information: journal name, year of publication, discipline, authorship (continent of university affiliation), type of paper (empirical or theoretical/conceptual), topic, the presence of an implicit or explicit chatbot definition, the original definition proposed by the authors, the terminology used to describe chatbots, the terminology used to describe the process of human-machine exchanges (e.g. dialogue, conversation, interaction), the conceptual foundations and theories, the perspectives addressed in the paper and the implications of the findings. For empirical papers, the methodology (quantitative, qualitative or mixed methods) and the dialogue metrics were also coded. Papers dealing with the development of instruments were also coded as empirical. Additionally, the presence of any ethical discussion was recorded. All this information was identified and then organized on a spreadsheet to enable comparison across studies and the translation of findings into higher-order interpretations. The disciplinary field was determined by the journal name and followed classification used by Statistics Finland (2018). An inductive approach was used since there is no list of predefined codes for the terminology used for chatbots, the terminology used to describe human-machine exchanges, the overall topic of the paper, the definitions of chats, theories and conceptual frameworks, metrics to measure chatbot functionality and the overall perspective discussed in the paper and its implications (Strauss and Corbin, 1990); codes were created while reading the articles. First, all terminologies, topics, theories, metrics, perspectives and implications as discussed by the original authors were recorded. Next, patterns of similarities across the 
$\mathrm{JCOM}$ 24,4

\section{4}

Figure 1.

Number of articles on chatbot-human exchanges by year codes were examined and grouped into macro-categories. Initial codes were revised on the spreadsheet to reflect the new categorization.

Perspectives were addressed based on the point of view underlined by scholars in the discussion and conclusion sections of the article; for example, if articles were about a chatbot's implication for marketing, the article was coded as "marketing perspective". Similarly, for the implications, all mentioned implications were recorded first and later grouped into macro-categories based on similarities of the conclusions and the reflections offered. The data on implications were divided into six macro-categories (see Appendix 1). For the definition of chatbots, all explicit and implicit definitions were copied and pasted and later thematically analysed to identify the main characteristics, descriptors and underlined capabilities. Constant consensus meetings of all researchers established the data extraction stage and the codes used in the analysis of publications.

An intercoder reliability test was performed on a random sample of seven articles on the latent variables. The first intercoder reliability test was low, and it was discovered that the descriptions of the codes, especially those regarding topics and implications, were too extensive and partly overlapping. The protocol was revised and further limited. After this, the articles were coded again and a new intercoder reliability test on another seven articles was performed. Results of this second check showed a good level of reliability. The level of intercoder agreement was $94.8 \%$ in every category.

\section{Results}

The analysis revealed that 62 papers were published in 40 different journals (see Appendix 1) and that the majority of the scientific papers were published in recent years (2015-2019), with a peak in 2019; in fact, by the fall of this year, 19 articles had already been published. However, an interesting note is that the first article was published in 1999 (see Figure 1).

In recent years (2015-2019), the most prominent disciplines contributing to chatbot research were computer and information science $(N=17)$, psychology $(N=14)$, general communication $(N=8)$ and marketing and management $(N=7)$. This indicates a lively and clearly diverse range of contributions to chatbot research. The largest group of researchers contributing to chatbots knowledge are affiliated with North American universities $(N=49)$, followed by European $(N=34)$ and Asian universities $(N=23)$. Fewer contributions come from scholars in Oceania $(N=7)$, South America $(N=1)$ and Africa $(N=1)[1]$.

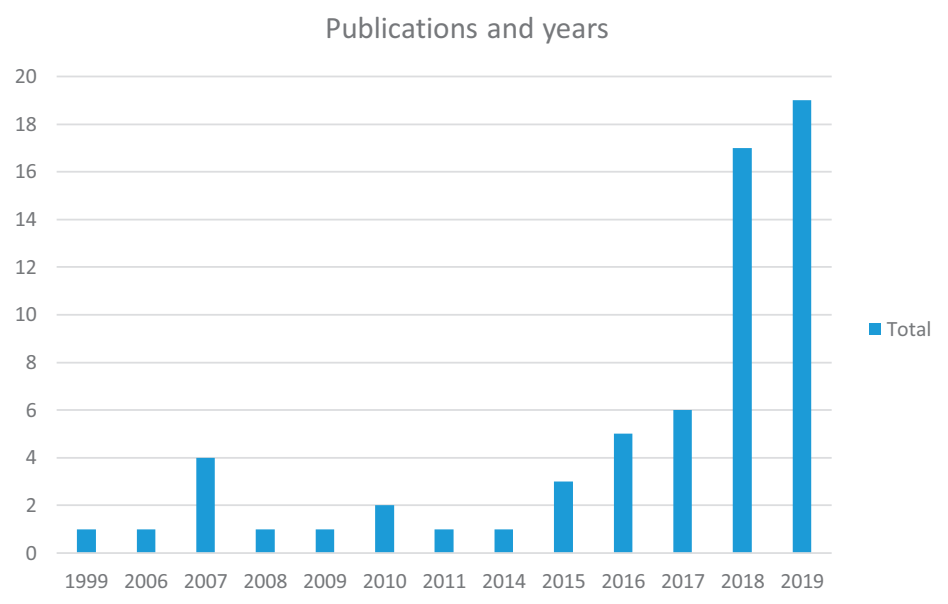


Publications dealt with a variety of topics (see Appendix 1), but the most important research topic was clearly chatbot development $(N=28)$. Testing chatbot language and conversation skills $(N=14)$ and how chatbots affect and are used in organizations $(N=14)$ were equally discussed. Chatbot as learning tools $(N=4)$ and troubleshooting chatbots $(N=2)$ were less relevant topics. This result is not surprising, given that the majority of the publications were in the computer science and engineering disciplines.

$R Q 1$. How are chatbots defined and used in the context of organization-stakeholder interactions?

To identify the preferred terminology for this technology, their capabilities and affordances, an inductive approach was used and all terms were recorded as well as all statements describing what they are, what they can do and any characteristic feature. This data were then grouped into three categories: terminology, descriptors and capabilities (see Figure 2). To illustrate how chatbots are defined and used in the extant literature, an onion structure was created. At the centre of the onion, the terminology recorded from the analysis is shown; in the next layer, the primary descriptors explicating the main characteristics of the technology are shown, and the last layer represents its capabilities based on previous academic literature on capabilities and affordances applied to the context of chatbots. It is important to note that elements in each layer can be combined differently and often with more than two features at once.

\section{Terminology}

The analysis indicated that articles used a variety of terms to define chatbots, often even interchangeably. For most, chatbot or chat bot or chatter bot were the preferred terms $(N=61)$, followed by agent $(N=21)$, $\operatorname{robot}(N=21)$, (conversational) bot $(N=10)$ and virtual

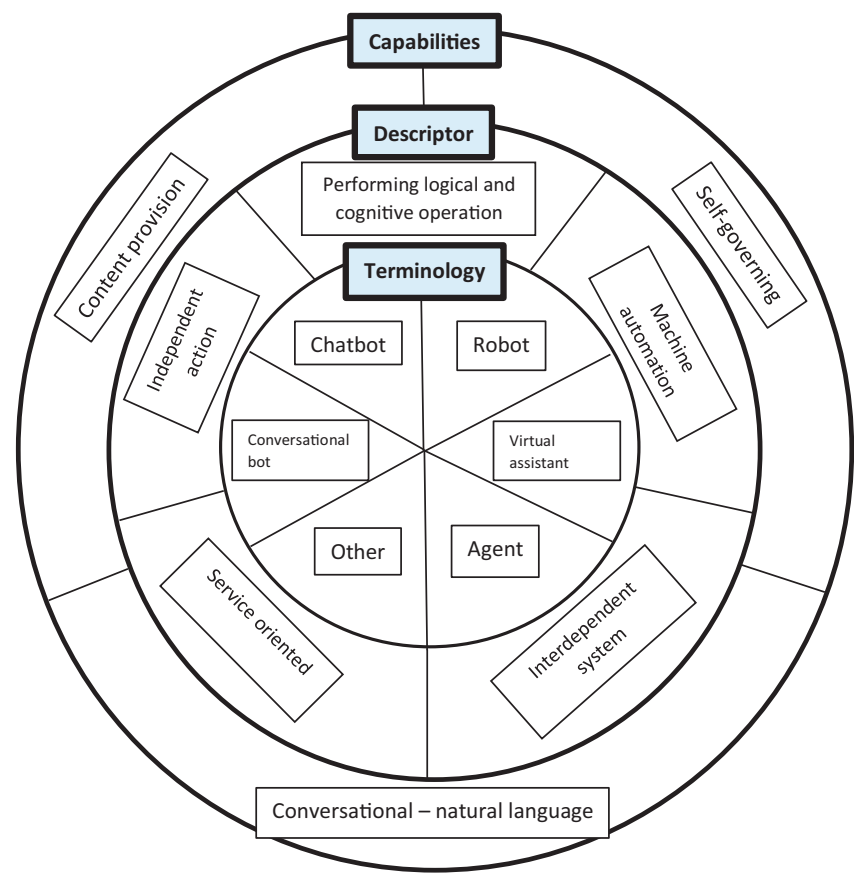

Figure 2.

Definitions of chatbots - terminology, descriptors and capabilities 
$\mathrm{JCOM}$ 24,4

\section{6}

assistant $(N=2)$ [2]. Just 29 papers out of 62 offered an explicit definition of chatbot. The definitions of chatbot varied but not significantly (Figure 2). Some definitions compared these technologies to IM Bots or content-driven bots. Others described them as forms of robots particularly in those articles where the preferred terminology was either chat robots, conversational service robots or a specific type of social robot. When scholars used the term agent, the definitions tended to focus on specific characteristics, such as being conversational, a machine, an artificial intelligence or a virtual process. Finally, some definitions of chatbots emphasised dialogue or machine conversation systems, chat services and communication programmes as main features.

We next analysed the terminology used in the literature to describe the context of usage, basically the material agency of chatbots in the context of organization-stakeholder interactions, that is, "the capacity for nonhuman entities to act on their own, apart from human intervention" (Leonardi, 2011, p. 148). Similarly, as for the actual naming of this technology, scholars have used a variety of different terms even in the same paper, thus we recorded all first-time appearances in the paper. Interaction $(N=53)$ was the most-used term to describe organization-stakeholder interactions via chatbots, followed by conversation $(N=38)$ and dialogue $(N=18)$. Discussion $(N=3)$, discourse $(N=2)$ and relationship $(N=1)$ were used marginally. It should be noted that in most of this literature, the concept of dialogue refers to the sequence of conversational turns that a machine, in this case chatbot, takes to reply to an inquiry (Jurafsky and Martin, 2019). Scholars using the term "dialogue" were thus particularly keen in studying the sequence of conversational turns in specific stakeholder interactions, rather than the orientation of communication towards dialogue. Overall, the top three terms for describing organization-stakeholder interactions via chatbots were expected since a large number of the articles were about developing and/or testing the humanness of this technology through measures of conversation skills and abilities that showcase particular types of material agency.

\section{Descriptors}

Looking closely at the main features that characterize these technologies, chatbots were primarily described as service-oriented, performing logical and cognitive operations, interdependent systems, machine automation and independent actors (see Table 1). Service-oriented chatbots were described as service providers trying to solve users' problems and respond to users' service needs. Chatbots that were described as performing logical and cognitive operations were based on coded or machine learning algorithms. These algorithms support logical and cognitive operations, for example, by selecting suitable answers and expressions to users' questions and comments. Chatbots with interdependent systems were described as relying on customer interaction history and able to process such information in their answering by using natural language. Machine automation was used to emphasise the mechanical performativity of chatbots and the technical features that allow chatbots to answer user questions without human help. Finally, several features pointed to independent action of chatbots, essentially underlining more social skills than technological ones, like reasoning and making decisions when the chatbot interacts or communicates with a user.

\section{Capabilities}

Based on descriptors, we identified the different capabilities of chatbots. Chatbots were seen to possess content provision, conversational and self-governing capabilities. Content provision means that the chatbot is providing content in the form of textual or verbal information and delivering knowledge, for instance, offering information to customers, answering questions and so on. The conversational capability arises from the chatbot's 


Descriptor
Service-oriented
Performing logical and
cognitive operations

Interdependent system

Machine automation

Independent action
Chatbot is based on coded or machine learning algorithms that support logical and cognitive activities
Chatbot processes previous interactions and uses such knowledge to answer automatically using natural language

Chatbot interacts mechanically with users without human help

Chatbot makes decisions independently without human help
Quote

"Machine agents serving as natural language user interfaces to data and service providers, typically in the context of messaging applications" (Følstad and Brandtzaeg, 2017, p. 38)

"Driven by algorithms of varying complexity, chat bots respond to users' messages by selecting the appropriate expression from preprogramed schemas, or in the case of emerging bots, through the use of adaptive machine learning algorithms. Chat bots can approximate a lively conversation with a human user, giving the illusion of intelligence or humanness" (Neff and Nagy, 2016, p. 4915.)

"In chatbots, the system is fed with natural language data on historical customer interaction, which is processed by an intelligent system that learns to automatically suggest answers back to the customer in text format" (Riikkinen et al., 2018, p. 1146)

"[A chatbot is] an automatic system capable of emulating a human being in a dialog with another person, in order to complete a specific task" (Griol et al., 2019, p. 28)

"[A chat bot has] autonomous and adaptable interfaces that interact, communicate and deliver service to an organisations' customers" (Wirtz et al., 2018, p. 909)

\section{Agents in organization- stakeholder interactions}

Table 1.

Descriptions of chatbots

purpose to interact and engage in conversations. Interaction can happen via text or voice through talking heads or embodied animated avatars. The conversational capability is a particularly relevant feature of chatbots as it differentiates them from other technologies by indicating how close to human-like the communication exchange is between chatbots and humans. Another important capability is the autonomy of the technology, what we called the self-governing capability. The fact that this technology can make choices in what and how to answer and can learn from previous conversational exchanges is seen as a specific element of the chatbot.

RQ2. What are the major theoretical and methodological approaches used to study chatbots' communicative exchanges?

Eleven different conceptual foundations were recorded from the analysed articles, and these pertain the disciplinary domains of computer science $(N=16)$, engineering $(N=15)$, psychology $(N=15)$, computer-mediated communication $(N=15)$, sociology $(N=12)$, linguistics $(N=11)$, information technology $(N=7)$, organizational studies $(N=6)$, philosophy $(N=3)$, general communication $(N=3)$ and mass communication $(N=2)$. Ten 
$\mathrm{JCOM}$ 24,4

\section{8}

articles did not include any specific conceptual foundation as they did not refer to any model, framework or academic study that was possible to track back to a research field [3].

With respect to the theories, more than half of papers $(N=43)$ did not mention any theory despite reviewing early studies, existing models and empirical tests. In the remaining papers, 25 different theories were identified but none of them clearly rose above the others. Role theory was the most common $(N=7)$, followed by media equation theory $(N=5)$, unified theory of technology acceptance $(N=5)$, social information processing theory $(N=4)$, theory of relational coordination $(N=3)$ and innovation diffusion theory $(N=3)$.

Most articles were empirical studies $(N=43)$; among those, quantitative research methods were employed in 36 papers. Only two papers employed a qualitative approach. Five papers used mixed methods. All metrics measuring the quality of chatbot-stakeholder interactions were also systematically recorded to better understand how the academic community assesses the level of technical development of specific chatbot software in addressing diverse interactions. The quality of chatbot-stakeholder interactions was measured in 18 articles. In these articles, 81 discrete metrics were recorded (see Appendix 2) and later grouped into macro-categories on the grounds of their overall measurements. The most-cited metrics across all empirical studies measured perceived human feelings $(N=15)$ and chatbot interaction skills $(N=13)$. This is expected as the majority of the papers were technological and were interested in studying and measuring how individuals perceive the communicative interaction experience with the machine.

RQ3. What are the major perspectives and implications discussed in relation to chatbots?

With respect to the perspectives, eight main points of view were found (see Appendix 1 for an overview of the definitions of these perspectives). The top perspective was machine language $(N=23)$, followed by marketing $(N=11)$ and psychology $(N=6)$. The communication perspective was addressed in eight papers and organizational and stakeholder perspectives, respectively, in six papers. Ethical and managerial perspectives were addressed in only one paper. When cross-tabulating research topics and perspectives, the machine language perspective was used primarily in articles that studied topics such as chatbot development $(N=16)$ and testing chatbot language and conversational skills $(N=4)$. A marketing perspective was often taken in research that studied chatbot effects and usage in organizations $(N=6)$. A communication perspective was present in research that studied the development of chatbots $(N=3)$, testing chatbots language and conversation skills $(N=3)$ or chatbot usage in organizations $(N=2)$. But, the topic of chatbots as learning tools was only studied from a machine language or a psychological perspective, whereas troubleshooting chatbots was studied only from an organizational or an ethical perspective (see Figure 3).

The implications discussed in the reviewed literature primarily dealt with improving [the] chatbot as a tool $(N=24)$ and chatbot effects on stakeholder interactions, responses and engagement $(N=15)$ (see Figure 4$)$. When implications were cross-tabulated with disciplines, publications in computer and information science $(N=12)$ and psychology $(N=7)$ primarily focused on improving [the] chatbot as a tool. General communication papers addressed implications dealing with chatbot effects on stakeholder interactions, responses and engagement $(N=3)$, results of the research are improving chatbot as a tool $(N=2)$, and critical reflections on chatbot development $(N=2)$. Even though the industry is divided on the potentials and pitfalls of artificial intelligence, ethical discussions on the use of these technologies in organizational contexts are limited. Our analysis indicated that only four articles out of 62 included an ethical discussion regarding chatbots: two were in general communication articles, one in computer science and another in a marketing article. With regards to the topics, ethical issues were discussed when scholars addressed how chatbots affect and are used in organizational settings. 


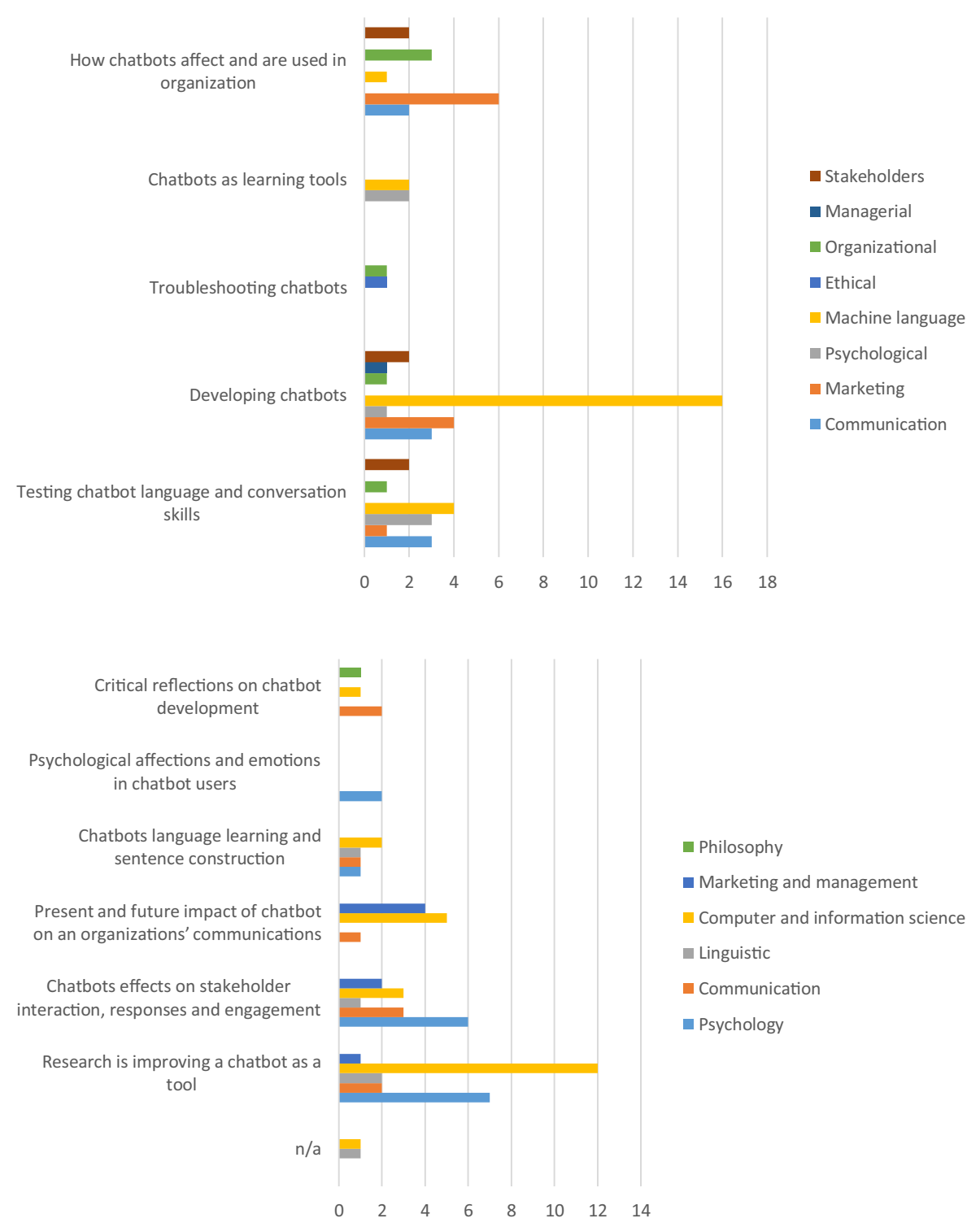

Agents in organizationstakeholder interactions

349

Figure 3.

Perspectives by research topics

\section{Discussion}

The results of this systematic literature review showed that chatbots have been studied in many different disciplines, but primarily in computer and information science $(N=24)$, psychology $(N=16)$ and general communication $(N=9)$. This versatile field of research might be one reason for the lack of a unified terminology to describe the chatbot phenomenon. The plethora of definitions indicates that there is a lively debate on the subject, but the lack of strong definition makes it difficult to compare research results and improve the quality of the research. 
$\mathrm{JCOM}$ 24,4

\section{0}

Contributions are diverse and geographically broad; however, a dominant Western perspective is visible, with most publications authored by European and North American scholars.

Looking closely at how chatbots have been studied, it is clear that the research community has identified several diverse descriptors for chatbots. For the most part, they focus on technological features and functional affordances. The most common feature is the natural language, meaning that chatbots employ a language that is natural or human-like when interacting with users (e.g., Zarouali et al., 2018; Holtgraves et al., 2007). Other important features are their ability to perform operations and to entertain users.

Taking into consideration these features, it is possible to outline two clear functional affordances in current research: chatbots can engage (emotional affordance) or assist (cognitive affordance) users. Engaging affordances are the capabilities of this technology to show emotions and feelings like a human in online interactions. This affordance was identified in those articles where scholars were primarily stressing that the chatbot was developed to engage users in conversations. Assisting affordances explained the processes and dynamics of the chatbot's systemic decision making during interactions. Here scholars focused on the technical, rational paths that the machine can take to talk or simulate conversations with humans. Both are relational affordances (Hutchby, 2001) as they describe how chatbots undertake a stakeholder communicative interaction, and represent two examples of latent capabilities emerging in a particular context (Rice et al., 2017).

Our analysis also shows that chatbots can possess three major capabilities: content provision, conversational and self-governing capabilities. Content provision is the capacity to detect, analyse and deliver tailored information. This is expressed as a functional affordance as it is a technical characteristic of the agent that allows it to respond to multiple and purposive communication requests. Conversational capability refers to the chatbot's capacity to be like a human in communicating and elaborating complex communicative interactions that extend beyond simply providing relevant content. This affordance is not an exclusive property of the chatbot, but it is the result of interactions between humans and chatbots since chatbots learn from communicatively interacting with humans and develop different strategies to cope with diverse scenarios. Hence, it can be considered a relational affordance (Hutchby, 2001). Finally, the self-governing capability refers to the level of independence of the agent in learning and adapting to different communicative situations. This is a material affordance as it is a property of the chatbot's algorithm to work on its own, to cognitively make decisions and so on. Chatbots offer a unique technological organizational asset for information processing, communication and knowledge management. These, it can be argued, represent elements of what Knight and Cavusgil (2004) claimed are the two major aspects of organizational capabilities. Specifically, chatbots are impacting and sometimes shifting the character of a business environment as they introduce new approaches and ways to handle, for instance, customer service. Because they are adaptive and intelligent systems, they can influence the strategic management of an organization in appropriately adapting, integrating, and re-configuring knowledge-based capabilities that may occur.

Hence, it becomes clear that chatbot affordances are much more sophisticated than those of other technologies that have been studied in public relations and corporate communication. For example, comparing chatbots to social messengers, messengers can foster strong one-onone connections with stakeholders (Tsai and Men, 2018), and chatbots can perform multiple conversations at the same time. The conversational capability also allows a chatbot to create engaging conversations without the assistance of a human community manager (MartinezLòpez et al., 2017). Nonetheless, caution should be used in concluding that chatbots can completely replace humans in online stakeholder conversations. Chatbots are still less human-like than humans (De Kleijn et al., 2019) and are unable to hold longer conversations or understand which direction the conversation is going. Chatbots are also missing context 
awareness (Chakrabarti and Luger, 2015), which clearly may become an issue because this negatively affects the user's experience.

Chatbot development relies heavily on artificial intelligence. Research is growing steadily in this regard. Improved interaction skills would increase the affordances of chatbots and make them more autonomous in a range of social interactions that support functions such as those of online sales persons, spokespersons or publicity agents. This is likely to happen, as the findings of this study show that current scholarship is highly focused on addressing human feelings and the chatbot's interaction skills.

Increasing agent interaction skills may thus lead to positive effects on stakeholder perceptions of organizations. Human-like interaction helps increase social relatedness, which has been shown to affect customer engagement and satisfaction (Kim and Drumwright, 2016) and create a positive correlation between online interactions and user engagement (Ji et al., 2017).

However, many questions remain. How can biases be prevented in chatbot learning. Is it ethical to try to replace humans with chatbots particularly when the decision tasks are highly sensitive? Whose is responsible for chatbot errors? There are only a few studies taking an ethical perspective on chatbots and more is needed to better grasp potential issues in chatbot adoption in organizational settings. Further developments of chatbots and artificial intelligence will show whether chatbots can or cannot respond to the challenge of becoming one of the most important interaction tools in organizations and actually help support stakeholder-organization relationships.

As a final point of reflection, even though chatbots are increasingly being adopted for diverse organization-stakeholder interactions, they are not much studied from organizational, stakeholder or corporate communication perspectives. Current studies focus on technical developments of chatbots and chatbot language and conversational skills. In these studies, communication is understood as a technical means of conveying words from/ to humans and not as a constitutive element or strategic resource in machine-human interactions. When chatbots are used to interact with stakeholders, they create specific types of dialogues with stakeholders, and engagement is one of their primary desired outcomes. Yet, the concept of engagement is understudied. These aspects have not yet been discussed in relation to chatbot capabilities to engage humans in conversations.

\section{Conclusions}

The purpose of this study was to examine chatbots in the context of online organizationstakeholder interactions and conduct a systematic literature review to identify what kinds of factors have been studied in the extant chatbot literature. Our findings showed that the chatbot is a new phenomenon as the majority of publications are rather recent (2015-2019). The reviewed literature is concerned for the most with investigating the machine language properties and the applicability of chatbot for marketing purposes. This systematic literature review points out that research has remained anchored at the micro level of understanding of the phenomenon, that is, the nature of chatbots, but has not yet taken into consideration the meso (organizational) or macro (societal) levels. Scholars have mostly been interested in studying the chatbot's interaction skills and developing chatbots, but in future studies, scholars should study chatbots and the impact of artificial intelligence on a larger scale. For instance, is development of AI and chatbots affecting organizational structures and the work content of employees? How are chatbots affecting the reputations of organizations? Is the use of chatbots reliable and accountable for corporate communication needs? Who is responsible for the decision making and communications of the machines?

This study has also revealed major research gaps, especially in corporate communication, which open avenues for future research. These appear to be the most important aspects to consider: 
$\mathrm{JCOM}$ 24,4
(1) There is a lack of organizational perspective in the research on chatbots. The whole idea of chatbots as smart agents affecting organizational boundaries, communication and storage (Carley, 2002) is missed, albeit very important. Artificial intelligence offers an opportunity to improve information delivery and information analysis (Pertucci, 2018, April 20), but more research is needed to understand how chatbots could be a useful tool for internal and external organizational communications. Future research could, for instance, look at specific chatbots capabilities and affordances in relation to internal communication processes, such as how chatbots could improve the flow of information and information retrieval in organizations. Developing and studying more chatbot capabilities and affordances can also inform managers and professionals on how such usage affects the organization's stakeholder engagement outcomes and what strategies to take on. New technologies like chatbots offer new opportunities for organizations to engage stakeholders, and recent research has begun to use the term "virtual engagement" to describe this phenomenon (Chewning, 2018). Yet, the concept of virtual engagement is understudied, but it could offer some interesting insights for the field of corporate communications and stakeholder engagement.

(2) There is a lack of a stakeholder perspective in the extant research of chatbots, that is, not much is known about how the end user feels about such interactions in situations and contexts of relevance for corporate communications. Few studies show a relevant difference in the quality of human-to-human interactions and those of human-to-chatbot interactions (Hill et al., 2015), but these are not enough. Scholarly research has mostly taken a technological perspective investigating chatbot interaction skills for the purpose of improving them, but the significance and importance of deploying chatbots on organization-stakeholder interactions or whether and how chatbots could affect stakeholder engagement have not yet been researched, and more is certainly called for, particularly studies to unveil if and how chatbots are capable of meeting stakeholder interaction expectations.

(3) There is almost no ethical discussion in the current chatbot research. This is problematic not just for the fact that these agents can develop deviant personalities and promote ideological answers not supporting an organization's goals, but it is academically problematic because all major theoretical foundations in corporate communication and related disciplines are basically short in explaining, describing and foreseeing the processes and outcomes of organization-stakeholder interactions via AI. To give an example, in public relations, the concept of dialogue (Kent and Taylor, 2002) is essentially challenged by the chatbot research definition of a dialogue. Dialogic theory is not suitable to explain an organization's ethical orientation in stakeholder communications via chatbots. We know little about whether and how ethical conversations with chatbots are, nor do we know much how to use chatbots in an ethical way to create mutually beneficial stakeholder interactions. More ethical discussion and research is called for.

In conclusion, future research studying chatbots from organizational, stakeholder and especially, a corporate communication perspective is of an impellent need. As chatbots are already part of organization-stakeholder interactions, future studies could clarify what specific affordances chatbots have that could serve different organizational and communication activities and explain the implications of using chatbots for organization-stakeholder interactions. 


\section{Limitations}

One of the primary limitations of this study is the number of articles reviewed. Because we were interested in analysing only academic research, we focused on academic peer-reviewed papers and excluded conference proceedings, books, book chapters and editorials, which may have offered other important and relevant reflections. Second, one of the goals of this review was to reflect on the status of knowledge on chatbot interactions with stakeholders from a corporate communication point of view, so the limited number of studies with a communication perspective (only seven), but not corporate communication, has shown that communication scholars still have much to contribute to the discussion of chatbot interactions.

\section{Notes}

1. Papers with multiple authors from different geographical locations were coded multiple times to represent geographically all contributors, thus the total number of authors recorded is greater than the total number of papers.

2. A paper could use more than one term. We recorded all first-time terms used in each paper, thus the total number of terms recorded is greater than the total number of papers.

3. A paper could use more than one conceptual foundation. We recorded all first-time conceptual foundations used in each paper; thus, the total number recorded is greater than the total number of papers.

\section{References}

Barad, K. (2003), "Posthumanist performativity: toward an understanding of how matter comes to matter", Signs, Vol. 28 No. 3, pp. 801-831.

Bell, M. and Pavitt, K. (1995), "The development of technological capabilities", in Ul Haque, I., Bell, M., Dahlmann, C., Lall, S. and Pavitt, K. (Eds), Trade, Technology and International Competitiveness, The World Bank, Washington, DC, pp. 69-102.

Bharadwaj, A.S. (2000), "A resource-based perspective on information technology capability and firm performance: an empirical investigation”, MIS Quarterly, Vol. 24 No. 1, pp. 169-196.

Bingjie, L. and Sundar, S.S. (2018), "Should machines express sympathy and empathy? Experiments with a health advised chatbot", Cyberpsychology, Behavior, and Social Networking, Vol. 21 No. 10, pp. 625-636.

Bourne, C. (2019), "AI cheerleaders: public relations, neoliberalism and artificial intelligence”, Public Relations Inquiry, Vol. 8 No. 2, pp. 109-125.

Carley, K.M. (2002), "Smart agents and organizations of the future", in Lievrouw, L. and Livingstone, S. (Eds), Handbook of New Media, Sage, London, pp. 206-220.

Chakrabarti, C. and Luger, G.F. (2015), "Artificial conversations for customer service chatter bots: architecture, algorithms, and evaluation metrics”, Expert Systems With Applications, Vol. 42 No. 20, pp. 6878-6897.

Chen, Y.-R.R. and Fu, J.S. (2016), "How to be heard on microblogs? Nonprofit organizations' follower networks and post features for information diffusion in China", Information, Communication and Society, Vol. 19 No. 7, pp. 978-993, doi: 10.1080/1369118X.2015.1086013.

Chewing, L.V. (2018), "Virtual engagement: a theoretical framework of affordances, networks and communication", In Johnston, K. and Taylor, M. (Eds), The Handbook of Communication Engagement, Wiley, New York, NY, pp. 439-452.

Conole, G. and Dyke, M. (2004), "What are the affordances of information and communication technologies?", ALT-J, Research in Learning Technology, Vol. 12, pp. 113-124.

\section{Agents in organization- stakeholder interactions}


$\mathrm{JCOM}$ 24,4

De Kleijn, R., Wijnen, M. and Poletiek, F. (2019), "The effect of context-dependent information and sentence constructions on perceived humanness of an agent in a Turing test", Knowledge-Based Systems, Vol. 162, pp. 794-799.

Duhé, S. (2015), "An overview of new media research in public relations journals from 1981 to 2014", Public Relations Review, Vol. 41 No. 2, pp. 153-169.

Dutrénit, G. (2004), "Building technological capabilities in latecomer firms: a review essay", Science Technology and Society, Vol. 9 No. 2, pp. 209-241.

Evans, S.A., Pearce, K.E., Vitak, J. and Treem, J.W. (2017), "Explicating affordances: a conceptual framework for understanding affordances in communication research", Journal of ComputerMediated Communication, Vol. 22 No. 1, pp. 35-52.

Faiola, A. and Matei, S.A. (2010), "Enhancing human-computer interaction design education: teaching affordance design for emerging mobile devices”, Int J Technol Des Educ, Vol. 20 No. 3, pp. 239-254.

Følstad, A. and Brandtzaeg, P. (2017), "Chatbots and the new world of HCl", Interactions, Vol. 24 No. 4, pp. 38-42.

Galloway, C. and Swiatek, L. (2018), "Public relations and artificial intelligence: it's not (just) about Robots", Public Relations Review, Vol. 44 No. 5, pp. 734-740.

Gao, Q., Sun, C. and Yang, C. (2014), "The influence of network structural properties on information dissemination power in microblogging systems", International Journal of Human-Computer Interaction, Vol. 30 No. 5, pp. 394-407.

Gilani, H. and Cunningham, L. (2017), "Employee branding and its influence on employee retention: a literature review", The Marketing Review, Vol. 17 No. 2, pp. 239-256.

Goh, O.S., Fung, C.C. and Depickere, A. (2008), "Domain knowledge query conversation bots in instant messaging (IM)", Knowledge-Based Systems, Vol. 21 No. 7, pp. 681-691.

Griol, D., Sanchis, A., Molina, J.M. and Callejas, Z. (2019), "Developing enhanced conversational agents for social virtual worlds", Neurocomputing, Vol. 354, pp. 27-40.

Grunig, J.E. and Huang, Y.H. (2000), "From organizational effectiveness to relationship indicators: antecedents of relatioships, public relations strategies, and relationship outcomes", in Ledingham, J.A. and Bruning, S.D. (Eds), Public Relations as Relationship Management, Erlbaum, Mahwah, NJ, pp. 23-58.

Guzman, A. and Lewis, S.C. (2020), "Artificial intelligence and communication: a human-machine communication research agenda”, New Media \& Society, Vol. 22 No. 1, pp. 70-86, doi: 10.1177/ 1461444819858691.

Hill, J., Ford, W.R. and Farreras, I.G. (2015), "Real conversations with artificial intelligence: a comparison between human-human online conversations and human-chatbot conversations", Computers in Human Behavior, Vol. 49, pp. 245-250.

Holtgraves, T.M., Ross, S.J., Wayward, C.R. and Han, T.L. (2007), "Perceiving artificial social agents", Computers in Human Behavior, Vol. 23 No. 5, pp. 2163-2174.

Huang, J. and Yang, A. (2015), "Implementing dialogic communication: a survey of IPR, PRSA, and IABC members", Public Relations Review, Vol. 41 No. 3, pp. 376-377.

Hutchby, I. (2001), “Technologies, texts and affordances”, Sociology, Vol. 35 No. 2, pp. 441-456, doi: 10. 1177/S0038038501000219.

Ji, Y.G., Li, C., North, M. and Liu, J. (2017), "Staking reputation on stakeholders: how does stakeholders' Facebook engagement help or ruin a company's reputation”, Public Relations Review, Vol. 43 No. 1, pp. 201-210.

Jurafsky, D. and Martin, J.H. (2019), Speech and Language Processing, Prentice-Hall, Upper Saddle River, NJ.

Kent, M.L. and Taylor, M. (2002), "Toward a dialogic theory of public relations”, Public Relations Review, Vol. 28, pp. 21-37. 
Kim, L. (1997), From Imitation to Innovation. The Dynamics of Korea's Technological Learning, Harvard Business School Press, Boston, MA.

Kim, E. and Drumwright, M. (2016), "Engaging consumers and building relationships in social media: how social relatedness influences intrinsic vs. extrinsic consumer motivation", Computer in Human Behavior, Vol. 63, pp. 970-979.

Knight, G.A. and Cavusgil, T.S. (2004), "Innovation, organizational capabilities, and the born-global firm”, Journal of International Business Studies, Vol. 35 No. 2, pp. 124-141.

Leonardi, P.M. (2011), "When flexible routines meet flexible technologies: affordance, constraint, and the implication of human and material agencies", MIS Quarterly, Vol. 35 No. 1, pp. 147-167.

Lievonen, M., Luoma-aho, V. and Bowden, J. (2018), "Negative engagement", in Johnston, K. and Taylor, M. (Eds), The Handbook of Communication Engagement, Wiley, New York, pp. 531-548.

Liu, L., Lee, M.K.O., Liu, R. and Chen, J. (2018), “Trust transfer in social media brand communities: the role of consumer engagement", International Journal of Information Management, Vol. 41, pp. 1-13.

Lock, I. (2019), "Explicating communicative organization-stakeholder relationships in the digital age: a systematic review and research agenda", Public Relations Review, Vol. 45 No. 3, 101829.

Majchrzak, A., Faraj, S., Kane, G.C. and Azad, B. (2013), "The contradictory influence of social media affordances on online communal knowledge sharing", Journal of Computer-Mediated Communication, Vol. 19, pp. 38-55.

Martìnez-Lòpez, F.J., Anaya-Sànchez, R., Molinillo, S., Aguilar-Illescas, R. and Esteban-Millat, I. (2017), "Consumer engagement in an online brand community", Electronic Commerce Research and Applications, Vol. 23, pp. 24-37.

McDougall, P., Shane, S. and Oviatt, B. (1994), "Explaining the formation of international new ventures: the limits of theories from international business research", Journal of Business Venturing, Vol. 9 No. 6, pp. 469-487.

Nass, C., Steuer, J. and Tauber, E.R. (1994), "Computers are social actors", Proceedings of the SIGCHI Conference on Human Factors in Computing Systems, ACM, New York, NY, pp. 72-78.

Navarro, C., Morena, A. and Al-Sumait, F. (2017), "Social media expectations between public relations professionals and their stakeholders: results of the ComCap study in Spain", Public Relations Review, Vol. 43 No. 4, pp. 700-708.

Neff, G. and Nagy, P. (2016), "Talking to bots: symbiotic agency and case of tay”, International Journal of Communication, Vol. 10, pp. 4915-4931.

Oxford Dictionary (2019), available at: https:/en.oxforddictionaries.com/definition/chatbot (accessed 25 February 2019).

Petrucci, A. (2018), "How artificial intelligence will impact corporate communications", Forbes, available at: https:/www.forbes.com/sites/forbescommunicationscouncil/2018/04/20/how-artificial-intelligencewill-impact-corporate-communications/\#213e78ef1dc6 (accessed 21 February 2019).

Pickering, A. (1995), The Mangle of Practice: Time, Agency, and Science, University of Chicago Press, Chicago.

Rice, R.E., Evans, S.K., Pearce, K.E., Sivunen, A., Vitak, J. and Treem, J.W. (2017), "Organizational media affordances: operationalization and associations with media use", Journal of Communication, Vol. 67 No. 1, pp. 106-130.

Riikkinen, M., Saarijärvi, H., Sarlin, P. and Lähteenmäki, I. (2018), "Using artificial intelligence to create value in insurance", International Journal of Bank Marketing, Vol. 36 No. 6, pp. $1145-1168$.

Rybalko, S. and Seltzer, T. (2010), "Dialogic communication in 140 characters or less: how Fortune 500 companies engage stakeholders using Twitter", Public Relations Review, Vol. 36 No. 4, pp. 336-341.

Simon, F. and Tossan, V. (2018), "Does brand-consumer social sharing matter? A relational framework of customer engagement to brand-hosted social media”, Journal of Business research, Vol. 85, pp. 175-184. 
$\mathrm{JCOM}$ 24,4

Statistics Finland (2018). "Fields of science 2010", available at: https:/www.stat.fi/en/luokitukset/ tieteenala/ (accessed 15 July 2019).

Stoeckly, E., Uebermickel, F. and Brenner, W. (2018), "Exploring affordances of slack integrations and their actualization within enterprises -towards an understanding of how chatbots create value", Proceedings of the 51st Hawaii International Conference on System Sciences, pp. 2016-2025.

Strauss, A. and Corbin, J.M. (1990), Basics of Qualitative Research: Grounded Theory Procedures and Techniques, Sage Publications, Inc, Thousand Oaks, CA.

Teece, D. and Pisano, G. (1994), "The dynamic capabilities of firms: an introduction", Industrial and Corporate Change, Vol. 3 No. 3, pp. 537-556.

Tilson, D.J. (2017), "From the natural world to artificial intelligence: public relations as covenantal stewardship", in Brunner, B.R. (Ed.), The Moral Compass of Public Relations, Routledge, New York, NY, pp. 206-222.

Tsai, W.S. and Men, R.L. (2018), "Social messengers as the new Frontier of organization-public engagement: a WeChat study", Public Relations Review, Vol. 44 No. 3, pp. 419-429.

Ullrich, D. and Smallwood, N. (2004), "Capitalizing on capabilities”, Harvard Business Review, Vol. 82 No. 6, pp. 2-11.

Wang, R., Kim, J., Xiao, A. and Jung, Y.J. (2016), "Networked narratives on Human of New York: a content analysis of social media engagement on Facebook", Computers in Human Behavior, Vol. 66, pp. 149-153.

Wang, X., Wang, C., Ding, Z., Zhu, M. and Huang, J. (2018), "Predicting the populatiry of topics based on user sentiment in migroblogging websites", Journal of Intelligent Information Systems, Vol. 5 No. 1, pp. 97-114.

Watkins, B.A. (2017), "Experimenting with dialogue on Twitter: an examination of the influence of the dialogic principles on engagement, interaction, and attitude", Public Relations Review, Vol. 43 No. 1, pp. 163-171.

Weizenbaum, J. (1966). "ELIZA - a computer program for the study of natural communication between man and machine", Communications of AMC, Vol. 9 No. 1, pp. 36-45.

White, C.L. Nielsen, A.E. and Valentini, C. (2017), "CSR research in the apparel industry: a quantitative and qualitative review of existing literature", Corporate Social Responsibility and Environmental Management, Vol. 24 No. 5, pp. 382-394.

Wirtz, J., Patterson, P.G., Kunz, W.H., Gruber, T., Lu, W.N., Paluch, S. and Martins, A. (2018), "Brave new world: service robots in front line", Journal on Service Management, Vol. 29 No. 5, pp. 907-931.

Ye, L. and Ki, E.-J. (2012), "The status of online public relations research: an analysis of published articles in 1992-2009", Journal of Public Relations Research, Vol. 24 No. 5, pp. 409-434.

Zarouali, B., Van den Broeck, E., Walrave, M. and Poels, K. (2018), "Predicting consumer responses to a chatbot on facebook", Cyberpsychology, Behavior, and Social Networking, Vol. 21 No. 8, pp. 491-497. 
Appendix 1

Agents in organizationstakeholder

Codes Disciplines

1 Psychology

2 Communication

$3 \quad$ Language and linguistic

4 Computer and information science

$5 \quad$ Business, marketing and management

$6 \quad$ Philosophy
Journals

Computers in Human Behavior interactions

Cyberpsychology, behaviour and social networking

Behavior Research Methods

Communication Research

Communication Studies

Public Relations Review

International Journal of Communication

Business and Professional Communication Quarterly

Journal of Communication

Interactions

Speech Communication

Language and Linguistics Compass

Text\&Talk

Journal of Pragmatics

Computer Speech and Language

Expert Systems with Applications

Journal of Information Science and Engineering

Future Generation Computer Systems

Library Hi Tech

Library Hi Tech News

Knowledge-Based Systems

BT Technology Journal

International Journal of Human-Computer Studies

Artificial Intelligence Review

Communication of the ACM

Ethics and Information Technology

The Knowledge Engineering Review

International Journal of Speech Technology

Neurocomputing

Computers and Graphics

Computers in Industry

Government Information Quarterly

International Journal of Medical Informatics

Journal on Service Management

Studia Universitatis "Vasile Goldis" Arad. Economics

Series

Education and Management Engineering

Journal of Business Research

International Journal of Bank Marketing

Management

Philosophical Investigations 
$\mathrm{JCOM}$

24,4

\section{Codes Topics}

1 Testing chatbot language and conversation skills

2 Developing chatbots

3 Troubleshooting chatbots

358

$5 \quad$ How chatbots affect and are used in organizations

$\begin{array}{ll}\text { Codes } & \text { Definition of chatbot } \\ 1 & \text { Yes } \\ 2 & \text { No }\end{array}$

Codes Implicit or explicit definition

1 Implicit

$2 \quad$ Explicit

$0 \mathrm{n} / \mathrm{a}$

Codes Terminology use

1 Chatbot or chat bot or chatter bot

2 Conversational bot

3 Robot

$4 \quad$ Agent

$5 \quad$ Virtual assistant

Codes Terminology used for human-machine exchanges

1 Dialogue

2 Conversation

3 Interaction

4 Discussion

$5 \quad$ Discourse

6 Relationship

Codes Conceptual foundations

1 Psychology

$2 \quad$ Linguistic

3 Communication

4 Mass Communication

Descriptors

Articles that are about testing chatbot's own conversation skills

Articles about development, new skills and usage possibilities of chatbots and also future aspects Articles about troubleshooting chatbots and chatbot usage in problem-solving and, e.g., vandalism online Articles about how chatbots are used by people in language learning (chatbot as a practice tool, learning and assessment tool)

Articles about how chatbots are useful and used in organizations, how chatbots are effecting on user engagement, communication quality, customer satisfaction and value creation 
$6 \quad$ Organization

7 Philosophy

$8 \quad$ Sociology

$9 \quad$ Information technology

10 Computer science

$11 \quad$ Engineering

\section{Codes Metrics}

1 Amount of words, messages and characters

2 Tone of conversation/interaction with the chatbot

3 Perceived feelings (conversation with the chatbot)

$4 \quad$ Grammatical structures

$5 \quad$ Chatbots interaction skills

$6 \quad$ Conversation style

Codes Methodology

1 Qualitative

$2 \quad$ Quantitative

$3 \quad$ Mixed methods

Codes Perspectives

1 Communication

$2 \quad$ Marketing

$3 \quad$ Psychological
$50=$ Media equation theory

$51=$ Social information processing theory

$52=$ Expectance violation theory

$53=$ Information theory

$54=$ Media Richness theory

$60=$ Theory of relational coordination

$70=$ Theory of intentionality

$71=$ Computational theory of mind

$80=$ Actor-network theory

$81=$ Role theory

$82=$ Innovation diffusion theory

$90=$ An unified theory of technology acceptance

$91=$ Heretical theory (Intelligent machinery, Alan

Turing)

Technical papers without actual theories that are about human-robot/human-chatbot interaction. E.g.,

Computers are Social Actors (CASA), Uncanny valley

Technical papers about developing, engineering, programming and coding Chatbots, AI devices and programmes (no actual theories mentioned) (e.g., NodeRED, SS-BED)

\section{Descriptions}

Amount of words or messages, shorthand or emoticons

In article has been accounted different tones of conversation. (e.g. polite, sincerity, profanity, message supportiveness and effectiveness, friendliness)

In article has studied perceived feeling after having a conversation with the chatbot (e.g. eeriness, feeling supported, likeability, attitudes, spine-tingling perception, sadness)

Dialogue metrics are grammatical structures (e.g. vocabulary range, spelling, nouns, verbs, typos) Article has studied different factors about interaction (e.g. agreement, accuracy, follow-up questions, conversation turns, cooperation of speakers, correction rate, response satisfaction, conversation ability and skills, used words and themes, sentences and phrases) Article has been studied conversation styles, like speed, habits, special features (quick, slow, mean answers)

\section{Descriptions}

Article has communication perspective (e.g. about human-chatbot interaction and cognitive interaction skills)

Article has marketing perspective (e.g. about customer service chatbots and consumer responses to chatbots Article has psychological perspective (e.g. humane attitude, emotions and feelings in interaction with chatbots)

\section{Agents in organization- stakeholder interactions}


JCOM

24,4

$\begin{array}{ll}5 & \text { Ethical } \\ 6 & \text { Organizational }\end{array}$

360

$2 \quad$ Chatbots effects on stakeholder interaction, responses and engagement

3 Present and future impact of chatbot on an organizations' communications

$4 \quad$ Chatbots language learning and sentence construction

$5 \quad$ Psychological affections and emotions in chatbot users

$6 \quad$ Critical reflections on chatbot development

Codes Ethical discussion

1 Yes

Table A1.
Articles perspective is about machine language and it's development (e.g. how chatbots functions or interaction skills are developed)

Article discusses ethical issues as result of chatbots' usage

Article deals with chatbot contributions to meeting organizational goals

Article discusses how managers deal with chatbots, their perspective on potential uses

Article focuses on how chatbots and their usage affect stakeholders and organization--stakeholder relationship

\section{Descriptions}

No clear implications is offered

Research has developed and improved chatbot and discovered new ways to use chatbot. Models are compared or systems and models are improved in the research. Especially development and opportunities of AI is important in improving chatbots

Chatbot's conversation skills affect to user engagement satisfaction, and users engage with chatbots as with other humans. Developing natural interaction and language are important goals

Chatbots affect organization's communication but chatbot's potential are still underutilized and chatbot's role is not yet very well known in organizations Chatbots can effect positively on language learning by increasing interest and affecting linguistic accuracy. Chatbot's sentence construction also affects perceived humanness

Users have reacted when chatbot have expressed sympathy or noticed that chatbot or system can actually talk

Implications are about abilities and inabilities of $\mathrm{AI}$ and chatbots. AI has developed Chatbot's features 
Appendix 2

Agents in organizationstakeholder

Codes Macro categories Metrics
interactions

1 Amount of words, messages and characters

$1=$ Word counts

$2=$ Message counts

$3=$ Type-token ratio (total number of different words)

$4=$ Shorthand

$5=$ Emoticons

$6=$ LIWC metrics (counts words)

2 Tone of conversation/interaction with the

$7=$ Profanity (reinaava) chatbot

$8=$ User attitude towards chatbot

$9=$ Message supportiveness

$10=$ Message effectiveness

$11=$ Sincerity

$12=$ Thoughtful (pleasantness)

$13=$ Polite (pleasantness)

$14=$ Pre-responsive (pleasantness)

$15=$ Friendly self-introduction

$16=$ Colloquial style

$17=$ Friendly farewell

3 Perceived feelings (conversation with the chatbot)

$18=$ Eeriness (fear)

$19=$ Belief in robotic feelings

$20=$ Felt supported

$21=$ Likeability

$22=$ Intelligence

$23=$ Attitude

$24=$ Novelty

$25=$ Spine-tingling perception

$26=$ Perceived interactivity

$27=$ Perceived contingency

$28=$ Perceived dialogue

$29=$ User engagement (level of absorption)

$30=$ Topic involvement

$31=$ Perceived sadness

$32=$ Perceived recognition

$33=$ Perceived understanding

$34=$ Belief in robotic intelligence

$35=$ User satisfaction

$4 \quad$ Grammatical structures

$36=$ Grammatical structures

$37=$ Vocabulary range

$38=$ Spelling

$39=$ Upper/lower case

$40=$ Nouns

$41=$ Pronouns

$42=$ Verbs

$43=$ Articles

$44=$ Question words

$45=$ Word order in statements

$46=$ Word order in questions

$47=$ Utterances

$48=$ Typos

$49=$ Capitalized words 
JCOM

24,4

Codes Macro categories Metrics

362

$5 \quad$ Chatbots interaction skills

6 Conversation style (speed, habits, special features)

$50=$ Percentage of follow-up questions

$51=$ Number of coherent conversation turns

$52=$ Percentage of successful resolutions

$53=$ Gricean maxims (how speakers act

cooperatively)

$54=$ Agreement

$55=$ Precision

$56=$ Recall

$57=$ Accuracy

$58=$ Task completion

$59=$ Use of context

$60=$ Correction rate

$61=$ Response satisfaction

$62=$ Conversation ability

$63=$ Skilled (conversational skill)

$64=$ Human (conversational skill)

$65=$ Engaging (conversational skill)

$66=$ Used words

$67=$ Used tones

$68=$ Themes

$69=$ Detecting emotions in textual dialogues

$70=$ Word -level features

$71=$ Phrase-level features

$72=$ Sentence level features

$73=$ Semantic-level features

$74=$ Turing test

$75=$ Quick answers

$76=$ Slow answers

$77=$ Mean answers

$78=$ Talks about self

$79=$ Questions about the agent

$7 \quad$ Chatbot usage

$80=$ Previous usage

81 = Power usage (attitude and use of technologies)

Table A2.

\section{Corresponding author}

Salla Syvänen can be contacted at: salla.k.syvanen@jyu.fi

For instructions on how to order reprints of this article, please visit our website:

www.emeraldgrouppublishing.com/licensing/reprints.htm

Or contact us for further details: permissions@emeraldinsight.com 